\title{
Integrierte Versorgung unter gemeindepsychiatrischer Steuerung - Pro
}

\author{
Integrated Health Care Managed by Community Psychiatric Services - Pro
}

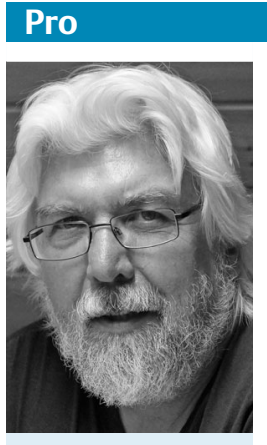

Reinhold Kilian
Deutschland gehört einerseits zu den Ländern mit den weltweit höchsten Ausgaben für die psychiatrische Versorgung, mit der größten Dichte an psychiatrischen Fachärzten, mit der höchsten Zahl an psychiatrischen

Krankenhausbetten, den meisten Psychiatrielehrstühlen, der besten Medikamentenverfügbarkeit und auch mit der umfassendsten Krankenversicherung, andererseits gleicht jedoch insbesondere das ambulante psychiatrische Versorgungssystem einem Flickenteppich, der weder dem Anspruch einer evidenzbasierten Gesundheitsversorgung noch dem in letzter Zeit zunehmend postulierten Anspruch nach gleichen Lebensverhältnissen gerecht wird [1, 2].

Praktisch zeigt sich diese Diskrepanz unter anderem darin, dass heute jeder von einer psychischen Erkrankung betroffene Mensch zwar an jedem Ort in Deutschland Anspruch auf ein Bett in einer psychiatrischen Klinik, auf die Behandlung durch einen Facharzt sowie auf das gesamte Spektrum derzeit am Markt verfügbarer psychopharmakologischer Substanzen hat, jedoch nicht auf evidenzbasierte ambulante psychosoziale Behandlungsformen, wie z.B. Home-Treatment, Krisenintervention, Soziotherapie, therapeutisches Case-Management, familienzentrierte Psychoedukation oder Supported employment [3]. Die regionale Verfügbarkeit dieser und eines breiten Spektrums von weiteren evidenzbasierten ambulanten psychosozialen Behandlungsangeboten hängt bis heute weitgehend von mehr oder weniger zufälligen Konstellationen aus dem Engagement von Laien, Politikern, Betroffenen und psychiatrischen Experten und den Aktivitäten von Kommunen, Gebietskörperschaften, Wohlfahrts- organisationen, freien Trägern, Krankenkassen usw. ab [4].

Die Ursachen der Diskrepanz zwischen einem, auch im internationalen Vergleich hervorragenden medizinischen und einem sowohl gemessen am wissenschaftlichen Kenntnisstand als auch am Anspruch der Wirtschaftlichkeit und der Versorgungsgerechtigkeit mangelhaften psychosozialen Versorgungssystem sind vielfältig. Einerseits liegen sie in einem seit den 1980er-Jahren zunehmend einseitig neurobiologisch dominierten Konzept psychischer Erkrankungen, welches psychosoziale Aspekte der Behandlung immer noch als der „originär medizinischen“, d. h. im Wesentlichen psychopharmakologischen, Behandlung, nachgeordnet betrachtet [5]. Andererseits liegen die Ursachen aber auch in einem immer noch durch die Ideen der Bismarck-Ära geprägten System der Organisation und der Finanzierung von Sozial- und Gesundheitsleistungen und den daraus resultierenden Zuständigkeits- und Interessenkonflikten. Die fragmentierende Wirkung der historisch gewachsenen Sozialsysteme wird im Fall der psychiatrischen Versorgung durch die föderale Zuweisung der Verantwortung an Länder und Kommunen verstärkt, die einerseits zwar durchaus sinnvoll ist, weil sie die für eine gemeindenahe psychiatrische Versorgung unabdingbare Berücksichtigung regionaler und lokaler Rahmenbedingungen (z.B. beim Wohn- und Arbeitsmarkt) gewährleistet, welche aber andererseits die Festlegung einheitlicher Struktur und Qualitätskriterien der psychiatrischen Versorgung verhindert.

Mit der Neufassung der $§ \S 140 f f$ des SGB $\mathrm{V}$ hat der Gesetzgeber die Problematik der historisch gewachsenen Fragmentierung der Organisation und Finanzierung psychiatrischer Versorgungsleistungen in Deutschland natürlich keineswegs gelöst. Er bietet jedoch eine Möglichkeit zur Überwindung der fachlich ungerechtfertigten Trennung medizinischer und psychosozialer Behandlungselemente und die Umsetzung von Behandlungskonzepten, die sich am Bedarf des Patienten und an der Evidenz für die Wirksamkeit von Behandlungsmaßnahmen orientieren und nicht daran, über welchen Kostenträger und nach welchen Modalitäten die einzelnen Leistungen abgerechnet werden können oder welche Institution oder Körperschaft für ihre Erbringung zuständig ist [6].

Bei der Frage der Trägerschaft für Verträge zur integrierten Versorgung wagt der Gesetzgeber einen, besonders für die psychiatrische Versorgung wichtigen Schritt in die Richtung der vorsichtigen Loslösung von einem einseitig neurobiologisch orientierten Krankheitsbild und den damit verbundenen Vorstellungen von einer „medizinisch/pharmakologischen“ als der einzigen adäquaten Gesundheitsversorgung. Damit eröffnet sich erstmals in der Geschichte der psychiatrischen Versorgung in Deutschland die Möglichkeit, dass Organisationen und Körperschaften, die sich über Jahrzehnte darum bemüht haben, in den Labyrinthen der Deutschen Sozialgesetzgebung nach Wegen für die Schaffung adäquater ambulanter psychosozialer Versorgungsstrukturen zu suchen [4], den Zugang zu einer zumindest theoretisch angemessenen Finanzierung ihrer Angebote durch die GKV erhalten [7].

Die Intention des Gesetzgebers bei dieser Öffnung des Zugangs zu einer GKV-Finanzierung lag natürlich keineswegs darin, die gemeindepsychiatrischen Träger für ihren hartnäckigen Einsatz für die Belange der sozialen Psychiatrie zu belohnen. Die Intention lag vielmehr eindeutig in der Schaffung von Anreizen für die Verbesserung der Effizienz der psychiatrischen Versorgung [6]. Obwohl die einfache Rechnung, dass ambulante psychiatrische Behandlungsformen in jedem Fall kostengünstiger sind als stationäre keineswegs immer zutrifft, legen gesundheitsökonomische Analysen eindeutig den Schluss nahe, dass sich durch eine stärkere Ambulantisierung das Kosten-Nutzenverhältnis der psychiatrischen Behand- 
lung auch aus der Perspektive der Patienten deutlich verbessern lässt [2].

Natürlich sollte man bei der Frage der Trägerschaft nicht vernachlässigen, dass sich auch psychiatrische Kliniken und Abteilungen ebenso wie niedergelassene Psychiater als „klassische“ Träger der psychiatrischen Versorgung vielfach um den Ausbau gemeindepsychiatrischer Versorgungsstrukturen bemüht, oder sich zumindest an diesen beteiligt haben und dass häufig erst die Kooperation mit diesen medizinischen Einrichtungen den gemeindepsychiatrischen Trägern die Etablierung ihrer Angebote ermöglicht hat $[8,9]$.

Legt man allerdings die Legitimität der Intention einer Verbesserung der Effizienz der psychiatrischen Versorgung zugrunde, spricht offensichtlich einiges dafür, die Steuerungsfunktion für Verträge zur integrierten psychiatrischen Versorgung auch in die Hände von Trägern zu legen, die kein ökonomisches Interesse daran haben, eine Umverteilung von Ressourcen vom stationären in den ambulanten Versorgungssektor zu verhindern. Die Träger der medizinisch-psychiatrischen Versorgung in Deutschland haben es in ihrer Gesamtheit über lange Zeit hinweg versäumt, ihre Monopolstellung für eine bessere Integration stationärer und ambulanter Bestandteile der psychiatrischen Versorgung zu nutzen. Die Geschichte der Einführung des neuen Entgeltsystems steht beispielhaft für dieses Versäumnis $[10,11]$.

Natürlich müssen auch die gemeindepsychiatrischen Träger ihre Fähigkeit und ihren Willen zu einer Neugestaltung der psychiatrischen Versorgung erst unter Beweis stellen. Der Wettbewerb zwischen medizinischen und gemeindepsychiatrischen Trägern um die Steuerungshoheit in der integrierten Versorgung könnte hier zumindest einen Anreiz für eine Entwicklung in die gewünschte Richtung bieten.

\section{Literatur}

1 Salize HJ, Rossler W, Becker T. Mental health care in Germany: current state and trends. Eur Arch Psychiatry Clin Neurosci 2007; 257: $92-103$

2 Salize H-J, Kilian R. Gesundheitsökonomie in der Psychiatrie. Konzepte, Methoden, Analysen. Stuttgart: Kohlhammer; 2010

3 Riedel-Heller SG, Becker T. [S3 guidelines on the psychosocial therapies of severe mental disorders]. Nervenarzt 2012; 83: 823-824

4 Greve N. Integration von Hilfen - Alltag der Gemeindepsychiatrie. In: Faulbaum-Decke W, Zechert C, Hrsg. Ambulant statt stationär. Psychiatrische Behandlung durch integrierte Versorgung. Köln: Psychiatrie Verlag; 2013: $117-126$

5 Priebe S, Burns T, Craig TK. The future of academic psychiatry may be social. Br J Psychiatry $2013 ; 202: 319-320$

Sie haben eine eigene Meinung zu diesem Thema? Dann schreiben Sie uns an: psychiat-praxis@thieme.de!
6 Roick C. Integrierte psychiatrische Versorgung aus der Sicht der Gesetzlichen Krankenversicherung. Nervenheilkunde 2013; 32: $264-269$

7 Faulbaum-Decke W. Gemeindepsychiatrie gestaltet Zukunft. Nervenheilkunde 2013; 32: $257-263$

8 Bock T, Lambert M. Hamburger Modell. Nervenheilkunde 2013; 32: 270-274

9 Roick C, Deister A, Zeichner D et al. [The regional budget for mental health care: a new approach to combine inpatient and outpatient care]. Psychiat Prax 2005; 32: 177 184

10 Kilian R. Wieviel Pep hat PEPP. Gesundheitsökonomische Auswirkungen pauschaler Entgeltsysteme für die stationäre psychiatrische Versorgung. Kerbe 2013; 31: $42-$ 44

11 Kunze H, Schepker R, Heinz A. Pauschalisierte Entgelte für Psychiatrie und Psychosomatik. Wohin kann der Weg gehen? Deutsches Ärzteblatt 2013; 110: 27 -28

\section{Korrespondenzadresse}

\section{Prof. Dr. rer. soc. Reinhold Kilian}

Klinik für Psychiatrie und Psychotherapie II, Sektion: Gesundheitsökonomie und Versorgungsforschung, Universität Ulm Ludwig-Heilmeyer-Straße 2

89312 Günzburg

reinhold.kilian@bkh-guenzburg.de

\section{Bibliografie}

Dol http://dx.doi.org/

10.1055/s-0034-1369953

Psychiat Prax 2014; 41: 182-183

(c) Georg Thieme Verlag KG

Stuttgart · New York

ISSN 0303-4259 